\section{FACTORY: A microcomputer program for simulation of production lines as a versatile tool for psychological experiments}

\author{
RAINER KÜNZEL \\ Ruhr-Universitat, Bochum, West Germany
}

FACTORY is a transaction-oriented computer simulation of a production line with quasi real-time characteristics. Because the program allows real-time operator interferences, a broad spectrum of human-machine interaction experiments is possible. The program is designed as a general tool in the sense that all parameters are accessible and changeable, so it can be shaped to different experimental characteristics.

The program allows the simulation of production lines that use a maximum of seven machines. If required, one of the machines may be switched on and off as a stand-in machine. The spatial design, the start of the production line, and the connections between the starting point and all machines are arbitrarily adjustable. The following characteristics of each machine are adjustable: speed, mean wear, and time used for maintenance and repair. The operator can obtain detailed numeric information about the condition of each machine at any time and shut down machines for maintenance.

Each machine has its own random generator that controls wear and breakdown of the machine. The risk of a breakdown depends on the degree of wear. An equally distributed random number (range 0 to 1 ) is produced after each production step and compared with the $n$th power of the scaled wear (range 0 to 1 ), where $n$ is a selectable coefficient. If scaled wear is greater than the random number, the machine breaks down during the production period of the next product equally distributed over the time.

This computer simulation has a number of possible applications: optimization problems, demonstrations, education, decision-making tasks, problem-solving experiments, sequential controlling tasks, risk-taking studies, and level of aspiration experiments. By choosing extreme parameter settings, one can solve general waiting-line problems. Krieger (1985), for example, used FACTORY to simulate the flow of drug-addicted patients within a health system of a medium-sized town by choosing a very long system-check interval and no wear, and by programming the rules of referral.

\section{Human Factors Example}

In this simulation, a human operator has to control a flexible robotic manufacturing system during an hour. The

The author's mailing address is: Ruhr-Universität Bochum, Psychologische Fakultät, Postfach 1021 48, D-4630 Bochum 1, Federal Republic of Germany. operator's task is to maximize the output of six automatic machines. He has to maintain the machines and avoid machine breakdowns. Within this context, two experimental conditions are compared: fully automated control and shutdown versus semiautomated human interference. The hypothesis is that human operators take more risks under full-information conditions than with reduced feedback.

FACTORY Setup: Six machines are positioned in two rows of three machines, parallel to each other on both sides of a central production line. Each machine is able to take over each of the six required steps of manufacturing. All machines have identical wear and speed $(6 \mathrm{sec}$ per step) characteristics. A machine performs an average of 100 steps without any needed maintenance of its mechanical parts. Time required to change parts is $90 \mathrm{sec}$ and for repair in case of a breakdown is $5 \mathrm{~min}$. Every $5 \mathrm{sec}$, a new product is carried to the starting point of the automated transport system. Since all machines perform the same steps of manufacturing, there is no prefixed course of production. An optimization algorithm allows for distribution of products to those machines with minimum waiting time. The operator can obtain numeric information about the condition of any particular machine and can maintain machines by pressing specific keys. The screen image of the simulated system is updated every second. The screen in the experimental condition looks like that in Figure 1.

Description. The simulation is based on the transactionoriented General-Purpose Simulator System (GPSS) (see Bobillier, Kahan, \& Probst, 1976; Schriber, 1974), which allows the simulation of waiting-line problems. Elements of GPSS are transactions, service stations, waiting queues, storages, and time- and situation-dependent actions. The FACTORY program uses a FORTRAN-subroutine library version of GPSS published by Schmidt (1978). FACTORY is written in Microsoft FORTRAN 3.31 and Microsoft Assembler MASM 3.0. It runs under the MSDOS 2.11 operating system. Target machines are IBMPCs or ATs with the ANSI.SYS device, APRICOT xi, and VICTOR 9000 , but because the program uses character graphics and no other machine-dependent characteristics, it would be easy to implement on any MS-DOS machine with different font and keyboard. Running FACTORY requires a minimum of $320 \mathrm{~KB}$ of memory.

The following characteristics of the production line can be set up in a file named FACTORY.INI: trace mode (on/off), upper time limit of the simulation (range 1 to $999999 \mathrm{sec}$ ), upper limit of products (range 1 to 999999), design of the instruments, exponent of the machine breakdown risk function, arrival intervals of products at the starting point of the transportation line, update interval of the instruments, number of production steps used for each single product, initial operating mode of the standin machine, $x y$ coordinates of the starting point of the production line and of all the machines, and possible des- 


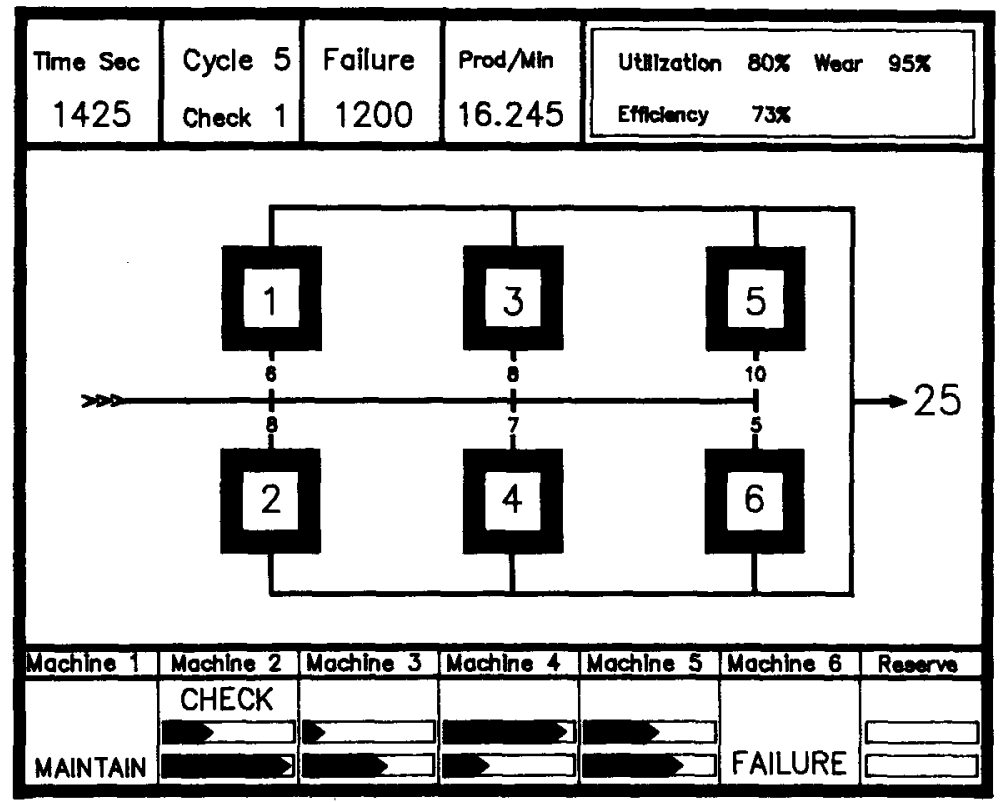

Figure 1. Screen setup of a human factors simulation (full-information condition).

tination and courses. Operating time, initial condition (new $=0$; out of order $=1000$ ), mean wear on the machine per product, loss of time per maintenance, and loss of time per repair are adjustable for each machine. The time of transit from one machine to another is calculated on a city-block metric design. If there is more than one possible destination point, the product is carried to the machine that will attend it first.

During operating time, a picture of the assembly line of the flexible manufacturing system is displayed and updated in fixed intervals, informing the user about elapsed time, arrival interval of products, interval of instruments updating, total time for breakdowns and repairs, output, and output per minute. Length of waiting line, wear condition, and residual machining time of the actual product in it are displayed for each machine. The screen image is loaded from a file and may be adjusted to the specific application with a text editor. On the operator's demand, wear condition and utilization and efficiency of each machine are shown in percent figures in a window for $5 \mathrm{sec}$.

At the end of simulation, some characteristics of each machine and of all machines together may be filed. These include break-off time of simulation, number of delivered products, idle time in breakdowns, idle time in repairs, number of extra readings of the instruments, and average condition at the point of shutdown for maintenance.

\section{Limitations}

Maximum number of machines is seven, and the number of products on the assembly line at the same time is limited to 200 . Both values may be increased according to available processor speed and memory of the target computer. The shortest time interval to which any process can be adjusted is $1 \mathrm{sec}$.

\section{Availability}

A copy of the source code, detailed instructions for installing and modifying the system, and a demonstration data set are available for IBM, APRICOT, or VICTOR formatted disk, by sending a floppy disk to the author.

\section{REFERENCES}

Bobillier, P. A., Kahan, B. C., Probst, A. R. (1976). Simulation with GPSS. Englewood Cliffs: Prentice-Hall.

KRIEGER, K. (1985). Computersimulation als Planungsmethode: Ein Vergleich des Ist-Zustandes mit den Zielvorstellungen der PsychiatrieEnquete im Bereich der Versorgung von Drogenabhangigen. Bochum: Diplomarbeit Ruhr-Universität.

SCHMIDT, B. (1978). GPSS-FORTRAN Version II: Einfühnung in die Simulation diskreter Systeme mit Hilfe eines FORTRANProgrammpaketes. Berlin: Springer.

SChriber, T. J. (1974). Simulation using GPSS. New York: Wiley.

(Revision accepted for publication October 3, 1986.) 